\title{
BM] open Design of an ecological momentary assessment study of exposure to radiofrequency electromagnetic fields and non-specific physical symptoms
}

\author{
Rik P Bogers, ${ }^{1}$ John F B Bolte, ${ }^{1}$ Jan H Houtveen, ${ }^{2}$ Erik Lebret, ${ }^{1,3}$ Rob T van Strien, ${ }^{4}$ \\ C Maarten A Schipper, ${ }^{1}$ Mehdi Alkadhimi, ${ }^{1}$ Christos Baliatsas, ${ }^{1,3}$ Irene van Kamp ${ }^{1}$
}

To cite: Bogers RP, Bolte JFB, Houtveen JH, et al. Design of an ecological momentary assessment study of exposure to radiofrequency electromagnetic fields and non-specific physical symptoms. BMJ Open 2013;3:e002933. doi:10.1136/bmjopen-2013002933

- Prepublication history and additional material for this paper is available online. To view these files please visit the journal online (http://dx.doi.org/10.1136/ bmjopen-2013-002933).

Received 22 March 2013 Revised 8 May 2013 Accepted 8 May 2013

${ }^{1}$ National Institute for Public Health and the Environment, Bilthoven, The Netherlands ${ }^{2}$ Department of Clinical and Health Psychology, Faculty of Social Sciences, Utrecht University, Utrecht, The Netherlands

${ }^{3}$ Institute for Risk Assessment Sciences (IRAS), Utrecht University, Utrecht,

The Netherlands

${ }^{4}$ Division of Environment and Health, Municipal Health

Services Amsterdam,

Amsterdam, The Netherlands

Correspondence to

Dr R P Bogers;

rik.bogers@rivm.nl

\section{ABSTRACT}

Introduction: Idiopathic Environmental Intolerance (IEI) attributed to electromagnetic fields (EMF) refers to self-reported sensitivity mainly characterised by the attribution of non-specific physical symptoms to lowlevel EMF exposure emitted from sources such as mobile phones. Scientific studies have not provided evidence for the existence of IEI-EMF, but these studies did not resemble the real-life situation or suffered from poor exposure characterisation and biased recall of health symptoms. To improve existing methods for the study of IEI-EMF, an Ecological Momentary

Assessment (EMA) study is designed.

Methods and analysis: The study is an EMA study in which respondents carry personal exposure metres (exposimeters) that measure radiofrequency (RF) EMF, with frequent assessment of health symptoms and perceived EMF exposure through electronic diary registration during five consecutive days. Participants will be a selection from an epidemiological study who report to be sensitive to RF EMF. The exposimeters measure electric field strength in 12 frequency bands. Diary questions include the occurrence and severity of 10 nonspecific physical symptoms, mood states and perceived exposure to (sources of) EMF. The relationship of actual and perceived EMF exposure and mood with non-specific physical symptoms will be analysed using multilevel regression analysis with time-shift models.

Discussion: The study has several advantages over previous studies, including assessment of personal EMF exposure and non-specific physical symptoms by an ecological method with a minimised chance of recall bias. The within-person design reduces confounding by time-stable factors (eg, personal characteristics). In the conduct of the study and the analysis and interpretation of its outcomes, some methodological issues including a high participant burden, reactivity, compliance to the study protocol and the potential of chance findings due to multiple statistical testing will be accounted for and limited as much as possible.

\section{INTRODUCTION}

Some people experience subjective health symptoms in the proximity of (specific sources

\section{ARTICLE SUMMARY}

Article focus

- The article describes the design of an Ecological Momentary Assessment (EMA) study to determine whether non-specific physical symptoms in persons who self-report to be sensitive to radiofrequency electromagnetic fields (RF EMF) can be explained by objectively measured exposure to RF EMF, or by psychological measures such as perceived exposure and mood.

Key message

- The EMA design of the study combines actual personal RF EMF exposure measurements with momentary-measured health symptoms, perceived RF EMF exposure and mood.

Strengths and limitations of this study

- Both personal EMF exposure and non-specific physical symptoms will be assessed by ecological methods with a minimised chance of recall bias.

- Issues to be aware of include a high participant burden, reactivity, compliance to the study proto$\mathrm{col}$ and the potential of chance findings due to multiple statistical testing.

or frequencies of) radiofrequency (RF) electromagnetic fields (EMF). Sources of RF $\mathrm{EMF}$ in the home environment include mobile or digital-enhanced cordless telecommunications (DECT) phones and their base stations, $\mathrm{WiFi}$, microwave ovens, television and radio transmitters. Although there is lack of a validated case definition, when an individual attributes his/her health symptoms to EMF exposure, this is mostly referred to as electromagnetic hypersensitivity. Owing to similarities with other (unproven) environmental intolerances, such as multiple chemical sensitivities, and because scientific evidence of a causal relationship between EMF exposure and symptoms is lacking, the WHO introduced the 
broader term Idiopathic Environmental Intolerance (IEI). ${ }^{1}$ When afflicted persons attribute their illness to EMF, it is referred to as IEI-EMF. The health symptoms of IEI-EMF are non-specific and differ from person to person. ${ }^{1}$ Frequently mentioned symptoms include fatigue, headaches, concentration problems, nervousness and tinnitus. ${ }^{2}$ IEI-EMF has been found to be associated with limitations in social and occupational functioning. ${ }^{3} 4$ The prevalence of IEI-EMF in the population is estimated to be $1.5-5 \%,{ }^{356}$ but a prevalence as high as $13 \%$ has also been reported. ${ }^{7}$ These differences are due to the population under study (Western countries vs Taiwan), and probably also the instruments or definitions that were used. ${ }^{8}$ For the Netherlands, an estimate of the prevalence of IEI-EMF is not yet available.

Scientific studies have not provided convincing evidence for the existence of a causal bioelectromagnetic mechanism for non-specific health symptoms. ${ }^{9}$ Alternative explanations for IEI highlight the role of psychological mechanisms, such as hyper vigilance to threat stimuli, attention bias and somatosensory amplification. ${ }^{10}$ For IEI-EMF, some findings suggest that nocebo responses account for the symptoms, ${ }^{9} 11$ in which concerns about a perceived harm precede the development of symptoms. Indeed, persons who suffer from IEI-EMF have relatively high levels of mental distress, anxiety, depression and worries about modern life. ${ }^{12-14}$ Several studies have demonstrated a relationship between negative affect and non-specific health symptoms. ${ }^{15}$

Most evidence for the lack of an association between EMF exposure and non-specific physical symptoms is derived from short-term provocation studies in the laboratory, which have been criticised because of their lack of internal and external (ecological) validity. Criticisms include that a visit to the laboratory may cause anxiety that influences the results, that EMF exposure in the experimental setting does not resemble real-life EMF exposure, and that follow-up times are insufficiently long to capture participants' responses. ${ }^{9}$ Observational studies are subject to other forms of bias due to errors in the recall of symptoms (recall bias) and in the assessment of EMF exposure. ${ }^{16}$

The limitations aforementioned can be solved by ecological momentary assessment (EMA). With EMA, the assessment is momentary, on the spot in real life, and captures life as it is lived. ${ }^{17} 18$ More precise and ecologically valid EMA measurement of personal RF EMF exposure can be performed with exposimeters. ${ }^{19}$ This produces more valid estimates than other methods, such as self-reported exposure, geo-coded distance from sources of RF EMF (eg, base stations) or spot measurements. ${ }^{20}$ Recall bias of symptoms can be minimised by using EMA diary methods with short-time frames instead of asking participants to retrospectively report (the usual frequency of) symptoms over a prolonged period. ${ }^{21}$

This article describes the design of an EMA study on the relationship between real-life measured and perceived exposure to RF EMF and the real-time experience of non-specific physical symptoms and mood in selfdeclared electrohypersensitive people. The study intends to minimise sources of bias by using exposimeters to estimate RF EMF exposure and real time on the spot assessment of symptoms.

\section{Objectives}

The key objective of the study is to determine whether in a period of a few days non-specific physical symptoms in persons who report to be sensitive to RF EMF can be explained by objectively measured exposure to RF EMF, or by psychological measures such as perceived exposure and mood. Secondary objectives are to study the manifestation of non-specific symptoms in terms of severity and duration of symptoms, the lag time-in hours to days-between exposure and the presentation of symptoms and to characterise RF EMF exposure of persons with IEI-EMF.

\section{METHODS AND ANALYSIS}

\section{Study design}

Epidemiological panel studies, which have similarities to EMA studies, have been described as "prospective studies that follow a usually small group of individuals intensively over a short time period [...] with the objective to study short-term effects of a time-varying environmental exposure." ${ }^{22}$ A main advantage of a panel design is the availability of measurements of exposure and health outcomes at an individual level. The current study is an EMA study in which, for five consecutive days, participants carry a measurement set consisting of an RF EMF personal exposure metre, a so-called exposimeter, a global positioning system (GPS) logger and an electronic diary. The electronic diary assesses health complaints, perceived exposure and mood. It has to be completed directly at frequent, random alarm cues, as this prevents both recall bias and possibly planned high exposures shortly before the time when filling the diary. This design allows for studying whether non-specific physical symptoms are preceded by exposure to EMF, using various latency times, and/or whether these symptoms are related to psychological variables such as perceived exposure and mood.

\section{Selection of study population}

Participants will be recruited from respondents of an existing epidemiological study (EMPHASIS) on nonspecific physical symptoms and their relation with model-estimated actual and perceived EMF exposure. ${ }^{23}$ This study included 6304 persons who were selected from 21 general practices throughout the Netherlands, varying in level of urbanisation and stratified according to the distance of their residences to a mobile telephone base station. The response rate to the written questionnaire was approximately $50 \% .^{24}$

Participants will be selected from the respondents to this survey based on self-reported sensitivity to EMF 
measured with a five-point scale. People who indicated to fully or partly agree with the statement "I am sensitive to antennas and devices using wireless communication (eg, for radio, television, mobile phones, wireless internet, etc)" and who gave their consent to use their address to reapproach will be invited by post to participate in the study. All study materials (diaries, exposimeters and instructions) will be delivered at the participants' homes, where they will be orally instructed about the study procedures.

\section{Electronic diaries}

Diary methods are considered suitable to examine selfreported events and experiences in their natural, spontaneous context. Benefits of diary methods are that bias in the recall of events and experiences is reduced because the time between the occurrence of an event or experience and its reporting is minimised. ${ }^{17}$ Diary methods can appropriately address the research question of what the correlates and antecedents are of within-person variability in daily experiences. ${ }^{17}$

The current study will use electronic diaries. Advantages of electronic diaries include a higher participant compliance than paper and pencil diaries, ${ }^{25}$ control of alarm cues and a detailed log file for compliance check.

For diary keeping we will use LG P-500 Optimus One smartphones running on Android 2.3. Because the study population will include persons with IEI-EMF, the phone operates in flight mode without a SIM card. A check of exposure to extremely low frequencies (Emdex Lite, Enertech Consultants, California, USA) and RF (EME Spy 121, Satimo, France) confirmed that the exposure from the smartphone was negligible, that is, below the detection limits.

Special software for the diaries was developed using Java (Android V.2.2 or higher). The diary programme is based on software written for Palm-OS personal digital assistants, which has been developed and used by Houtveen $e t a l .^{26} \mathrm{~A}$ sampling protocol with a mean interval of $2.5 \mathrm{~h}$ and random variation of $\pm 30 \mathrm{~min}$ will be used that continues from awakening till bedtime. This sampling scheme leads to approximately 8 alarms per day (based on a $16 \mathrm{~h}$ awakening period). Diary prompting will only be disabled during sleep, initiated by a button on the smartphone. The smartphone can be used as a morning alarm, and prompting continues after awakening. All unused buttons are blocked. Alarms without response are repeated (maximum 3 times with $10 \mathrm{~min}$ time intervals). The alarm software generates a log-file containing alarm and response times to be used for determination of the compliance. The questionnaire can be launched by a start button that is visible for 5 min after prompting. All questions are forced-choice, and are displayed as sequential screens on the smartphone. Participants will not be allowed to leaf through the present or previous diaries. The volume of the alarms is adjustable and there is the possibility to temporarily mute the alarm.

\section{Diary questionnaire}

The diary questionnaire consists of 32 items. In the morning and evening, five and eight additional questions are included, respectively. According to guidelines for diary questions, the items are short, simply worded, and try to mimic the participants' internal dialogue, for example, 'at the moment, I suffer from headache'.

\section{General health status and non-specific physical symptoms}

General health status will be assessed by using the first question from the RAND-36. ${ }^{27}$ Nine symptoms are selected that are most often reported by electro hypersensitive people according to studies in Switzerland ${ }^{2}$ and a survey among members of the Dutch Electrohypersensitivity Foundation. ${ }^{28}$ These symptoms comprise fatigue, distressed/nervous/tense feeling, concentration problems, tinnitus, dizziness or light-headedness, painful joints or muscles, skin problems, problems with vision, hearing or smell. In addition, one question asks for the symptom that the participant usually experiences and that is not in the prespecified list. For all symptoms the momentary experience is assessed ('at this moment, ...'). Response options range from 'not at all' to 'very much' on a five-point Likert response format with only the extremes labelled.

\section{Perceived exposure to (sources of) RF EMF, noise and air pollution}

Perceived exposure to RF EMF will be assessed both momentarily and for the interval between alarm cues, using the question 'At the moment/since the last alarm cue, I am exposed to radio frequent electromagnetic fields', with response options ranging from 'not at all' to 'very much' on a five-point scale with only the extremes labelled. Perceived exposure to specific sources of RF EMF is assessed by asking the participant to indicate which of the following sources mainly determined their exposure: mobile phone, DECT phone, WiFi, antennas for mobile telephony, radio or television masts, another source.

Momentary (but not for the interval between alarm cues) exposures to noise and air pollution are asked in a similar way.

\section{Environment}

In order to interpret the readings of the exposimeter, participants will be asked to indicate the kind of environment they were in during the interval between alarm cues. The environments included are at home inside, at home outside, elsewhere inside, elsewhere outside, on the road (on foot, by bike, car or public transport), in the city centre or a shopping area, in a residential or built-up area but not the centre, outside the built-up area (eg, in a rural area or in nature). All participants already completed a detailed time-activity questionnaire on their general behaviour in the EMPHASIS study. ${ }^{23}$ 
Self-reported use of mobile phone and DECT phone

Phone use will be estimated separately for mobile phones and DECT phones based on the question of how many minutes a person called in the interval between alarm cues: $1-5,5-10,10-20,20-30,30-60$ or longer than $60 \mathrm{~min}$. In addition, participants will be asked to register on a form their use of mobile and DECT phones.

\section{Mood}

The Profile of Mood States ${ }^{29}$ will be used to assess momentary (state) depression, vitality, anger and tension. Three items for each subscale will be used, as was carried out previously by Houtveen and van Doornen. ${ }^{30}$ The selected items were as follows: (1) depression: unhappy, sad, hopeless; (2) vitality: active, energetic, lively; (3) anger: angry, annoyed, moody and (4) tension: tense, nervous, anxious. Each item can be rated using a five-point Likert response format ranging from 'not at all' to 'very much' with only the extremes labelled.

\section{Additional questions}

After the morning alarm cue, the diary contains some questions about the duration and quality of sleep. Before the participants go to bed, questions are asked about use of medication to relieve their symptoms (mentioned in the diary), avoidance of sources of RF-EMF because of their symptoms, and whether the participants rested or took a nap during daytime.

\section{Personal RF exposure assessment}

Actual EMF exposure will be measured using EME-SPY 121 exposimeters (Satimo, Cortaboeuf, France) worn at the hip in a camera bag. As the maximum memory capacity of the exposimeter is 12540 sampling intervals and the study will last for $120 \mathrm{~h}$, the exposimeter will measure at an interval of $36 \mathrm{~s}$. The exposimeters measure the RF electric field strength in 12 frequency bands used for communication and broadcasting (see online supplementary appendix 1). Before the exposimeters are employed in the study, they will be calibrated according to a previously described method, ${ }^{19}$ modified in a way that the calibrations take place in an anechoic chamber instead of a Gigahertz Transverse Electromagnetic cell (GTEM).

The participants will be instructed to wear the exposimeters all day except during wet activities (eg, showering) and sports (to avoid damage due to shocks). During sleep, the exposimeters have to be placed adjacent to the bed within $50 \mathrm{~cm}$ from the head. The electronic diary contains an event button that can be used to indicate when the exposimeters are worn or taken off.

\section{GPS logger}

Participants will wear a GPS device at their left shoulder. The GPS logger geo-locates the personal RF EMF measurements and the data can be used to visualise the participants' location and measured EMF exposure on a Google Earth map. ${ }^{31} 32$ This visualisation can be used to interpret and check the quality of the EMF measurements.

\section{Data analysis}

Exposimeter data will be aggregated either over fixed time intervals or over intervals between random alarm cues, and for each interval the time weighted average, peak exposure, exposure above a certain threshold and rate of change can be calculated for all frequency bands separately and combined. The relationship of actual and perceived EMF exposure with non-specific physical symptoms will be analysed using multilevel regression analysis. The within-participant repeated measurements of actual and perceived EMF exposure and mood will be the firstlevel variables, which will be modelled as fixed effects; the second level will be the individuals and will be modelled as random effects. As time (hour of the day) is associated with symptom occurrence and severity (eg, for fatigue), this will be included in the model using a sinus-cosinus $24 \mathrm{~h}$ function to account for the diurnal pattern. Unique contributions of the various explanatory variables will be estimated in multivariate models, in which an intercept, $24 \mathrm{~h}$ time function, actual EMF exposure, perceived EMF exposure and mood will be included. Time-shift models with different lag times will be used to gain insight into the time delay between exposure and mood and the occurrence of symptoms. The time window of 5 days should be sufficient to capture the potential participants' response to EMF exposure, based on reported latencies between exposure and symptoms. ${ }^{2}$

A multilevel power analysis was performed to calculate the strength of the association between perceived EMF exposure and non-specific physical symptoms that could be detected with the repeated measurements multilevel analysis in a sample of 60 volunteers with each 40 (5 days with 8 measurements) observations. A significance level of $5 \%$ and a power of $80 \%$ were used. This method based on simulations has been described previously. ${ }^{33}$ No such calculation was made for actual EMF exposure because most evidence points towards the absence of an association with non-specific physical symptoms.

Input parameters for the power analysis came from a pilot study of four (not electrohypersensitive) master students (2 men and 2 women) who completed the diary questions for 2 weeks (unpublished data, National Institute for Public Health and the Environment 2012). Figure 1 illustrates how the power of the statistical analysis differs according to the magnitude of the regression coefficient. It can be seen that at a power of $80 \%$, the detectable regression coefficient is slightly over 1.5 , which corresponds to an increase of 1.5 on the sum of 10 symptoms (range $0-40$ ) at an increase of 1 in perceived exposure (range $0-4$ ) 


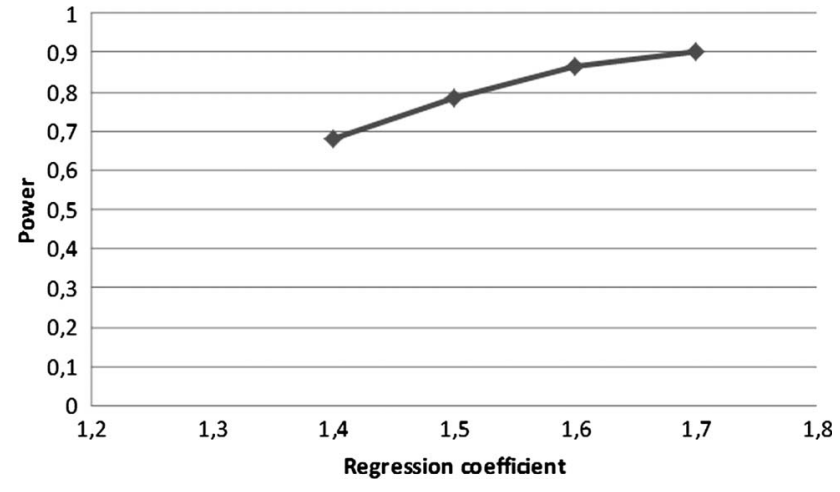

Figure 1 Power of the repeated measurements multilevel regression analysis as a function of the magnitude of the regression coefficient, $n=60$ persons with 40 repeated measurements.

\section{ETHICS AND DISSEMINATION}

A formal written inquiry, including a detailed description of the study protocol, was made at the Medical Ethics Committee of the University Medical Centre Utrecht to verify whether the study protocol should be tested within the framework of the Dutch Medical Research Involving Human Subjects Act. A formal advice was received in which the Committee indicated that the study was exempt from having to pass the full ethics testing procedure. Handling of personal data will comply with the Personal Data Protection Act (in Dutch: Wet bescherming persoonsgegevens (Wbp)). After removal of the identifying information, data will be stored on a part of the institute's server with limited access by specified employees.

Results of the study will be offered for publication in the international peer-reviewed literature and presented at (international) conferences. Further, results will be disseminated at a national level at meetings organised by The Netherlands Organisation for Health Research and Development (ZonMw). At these meetings representatives of IEI-EMF patient groups will also be reached.

\section{DISCUSSION}

The EMA design of this study is innovative as it combines actual exposure measurements with momentarymeasured health symptoms. The design aims to circumvent important limitations of previous studies into IEI-EMF, which can be summarised as biased recall of health outcomes, poor characterisation of individual exposure to EMF and experimental exposure conditions that substantially differ from the real-life situation (ie, low ecological validity). Another potential strength of the study is the simultaneous assessment of actual and perceived EMF exposure. In the multilevel regression analysis, independent contributions of these two types of exposure can be estimated, an approach similar to the EMPHASIS study. ${ }^{23}$ With respect to recall bias, it has been shown that especially in people with high levels of non-specific physical symptoms, less symptoms are reported when using momentary assessment methods compared with retrospective methods. ${ }^{34}$ Since persons with IEI-EMF typically report more symptoms than the general population, ${ }^{35}$ a symptom diary is more suitable to obtain a valid estimate than asking to report the usual or average number of symptoms retrospectively.

Regarding the EMA measurement of exposure, exposimeters are the method of choice to measure personal exposure compared with spot measurements, selfestimated exposure and exposure prediction models. ${ }^{20}$ Nevertheless, measurements of exposimeters also have their own limitations and are not always free of bias. To obtain valid measurements, it is important that the participants receive clear and standardised instructions about how to wear the metres. In this way, measurements by different participants will be harmonised and more comparable. ${ }^{19}$ Exposure from mobile phone use by the participants themselves will not be measured properly by the exposimeter because the exposimeters are designed to measure the far EMF field, that is, exposure from EMF sources further away. In the near field, the exposimeters are unable to correctly measure the exposure, resulting in overestimations or 'clipping' in which the maximum measurement value of $10 \mathrm{~V} / \mathrm{m}$ is registered. Therefore, mobile phone use during each $2.5 \mathrm{~h}$ interval between alarm cues is asked for in the diaries (although we are aware of the recall bias in the estimates of self-reported phone use) and participants are requested to register their use of mobile and DECT phones.

Another strength of using individual-level exposure and outcome data is that confounding by factors that remain stable over time is reduced because withinparticipant variation in exposure and manifestation of health symptoms are of primary interest. Thus, the analyses do not require adjustment for personal characteristics such as demographic factors and psychological traits. However, to detect possible interaction effects, for example, different associations in men and women, personal characteristics have to be taken into account. The multilevel regression analysis allows for such an investigation of cross-level interactions. As the study population may be a mix of 'truly' electrosensitive individuals and individuals in whom psychological mechanisms account for their symptoms, we will explore the applicability of statistical methods to study associations that differ between individuals (vector autoregressive models ${ }^{36}$ ).

Time-varying factors associated with EMF exposure ${ }^{32}$ that potentially affect symptom occurrence also need to be accounted for. Avoidance of EMF exposure after symptoms begin to develop is such a factor (which is asked for in the diary). Time-varying factors associated with symptoms but not with EMF exposure are no confounders, but can obscure relationships between exposure and symptoms. This is the reason a question about the use of medication to relieve symptoms and rest/ sleep during the day is included in the diary questionnaire. 
There are also limitations to the described study. Although the external (ecological) validity of the design is high, compared with double-blind trials such as provocation studies in the laboratory, the internal validity is lower. Further, the study involves relatively high costs in terms of the necessary equipment and work. The exposimeters are expensive and vulnerable to physical damage, and the electronic diaries are also costly compared with traditional paper and pencil diaries. Also, the programming of the diary software is very time consuming. As a result, only a limited number of participants can be included in the study. The burden for participants is quite high, since they have to carry the exposimeters and GPS loggers with them and are interrupted by the diary alarm cues several times a day. It is expected that only highly motivated persons will participate, but because the study is not intended to examine a representative sample of the population this is not considered a problem. Compliance to the study protocol may be difficult for some participants because they may find wearing the exposimeter awkward. As a result of participation in the study, the participants' attention to EMF exposure and physical symptoms may increase (reactivity). It can in principle not be excluded that the data are manipulated by placing the metres adjacent to a (assumed) source of EMF before an alarm cue is expected. To minimise the chance of such anticipation effects, the alarms are programmed at random intervals. Further, unusual exposure patterns can be checked for, although high and prolonged exposure peaks can be real and do not have to result from anticipation effects.

In the statistical analysis, false-positive associations due to multiple testing may arise. For EMF exposure, there is information about 12 frequency bands, which can be analysed separately and combined. Possible exposure metrics include time-weighted average, peak exposure, rate of change and exposure above a certain threshold. Since no biological mechanism is known that explains how EMF can affect health, in theory each combination of almost 50 combinations of frequency and exposure metrics can be relevant. To take into account the possibility of false-positive associations, the expected proportion of falsely rejected hypotheses will be controlled using a sequential Bonferroni-type procedure described by Benjamini and Hochberg. ${ }^{37}$ If associations with symptoms are detected, it is necessary to replicate the results to exclude chance findings. Also, it should be noted that significant relationships between RF-EMF exposure and symptoms not necessarily have to be causal relationships.

An issue that deserves attention is the selection of the study population. Since no objective diagnostic tool for IEI-EMF exists, the only selection criterion for inclusion in the study is self-reported sensitivity to RF EMF. No further selection will be made based on the occurrence of nonspecific physical symptoms as this is the outcome variable of the study. Also, no exclusions will be made based on psychological variables. The reason for this is that IEI-EMF often goes together with psychological problems, such as depression and anxiety, ${ }^{12}$ and especially in the most sensitive psychiatric morbidity has been reported to be high. ${ }^{7}$ Therefore, by excluding persons based on psychological characteristics the study population of interest may be missed. Somatic (chronic) diseases will not be excluded either, as a considerable part of persons having IEI-EMF suffer from chronic diseases, ${ }^{35}$ and at this stage it is not possible to decide on theoretical grounds which medical conditions should be included or excluded.

A control group is not deemed necessary, since in the longitudinal study design each participant acts as their own control. Moreover, if there are associations between RF EMF exposure and symptoms, this will be most likely in persons who report to be sensitive to RF EMF, and statistical power will be enhanced by focusing on this group. A limiting factor of this approach is that we cannot verify if persons with IEI-EMF are more sensitive to EMF than controls.

In summary, this is the first time that actual and perceived exposure and possibly explanatory variables are combined to such extent in an IEI-EMF sample. The main strengths of the study described in this article are an accurate assessment of EMF exposure and non-specific physical symptoms using an EMA methodology, and elimination of confounding by personal characteristics as a result of the within-person design. The methodological issues aforementioned will be accounted for and limited as much as possible in the conduct of the study as well as the analysis and interpretation of its outcomes.

Contributors RPB conceived the study, participated in its design and coordination, and drafted the manuscript. JFBB, JHH, RTVS, CAMS and CB participated in the study design and helped to revise the manuscript critically for important intellectual content. EL conceived the study, participated in the study design and helped to revise the manuscript critically for important intellectual content. MA programmed the electronic diary software and helped to revise the manuscript critically for important intellectual content. IVK participated in the study design, permitted access to the EMPHASIS dataset and helped to revise the manuscript critically for important intellectual content. All authors read and approved the final manuscript.

Funding This work is financially supported by The Netherlands Organisation for Health Research and Development (ZonMw), grant number 85600005.

Competing interests None.

Ethics approval University Medical Centre Utrecht.

Provenance and peer review Not commissioned; externally peer reviewed.

Open Access This is an Open Access article distributed in accordance with the Creative Commons Attribution Non Commercial (CC BY-NC 3.0) license, which permits others to distribute, remix, adapt, build upon this work noncommercially, and license their derivative works on different terms, provided the original work is properly cited and the use is non-commercial. See: http:// creativecommons.org/licenses/by-nc/3.0/

\section{REFERENCES}

1. World Health Organization. Electromagnetic hypersensitivity. WHO factsheet no. 296. World Health Organization, 2005.

2. Röösli M, Moser M, Baldinini $Y$, et al. Symptoms of ill health ascribed to electromagnetic field exposure-a questionnaire survey. Int J Hyg Environ Health 2004;207:141-50.

3. Hillert L, Berglind N, Arnetz BB, et al. Prevalence of self-reported hypersensitivity to electric or magnetic fields in a population-based questionnaire survey. Scand J Work Environ Health 2002;28:33-41. 
4. Kato $Y$, Johansson O. Reported functional impairments of electrohypersensitive Japanese: a questionnaire survey. Pathophysiology 2012;19:95-100. doi:10.1016/j. pathophys.2012.02.002

5. Levallois P, Neutra R, Lee G, et al. Study of self-reported hypersensitivity to electromagnetic fields in California. Environ Health Perspect 2002;110(Suppl 4):619-23.

6. Schreier N, Huss A, Roosli M. The prevalence of symptoms attributed to electromagnetic field exposure: a cross-sectional representative survey in Switzerland. Soz Praventivmed 2006;51:202-9.

7. Meg Tseng MC, Lin YP, Cheng TJ. Prevalence and psychiatric comorbidity of self-reported electromagnetic field sensitivity in Taiwan: a population-based study. J Formos Med Assoc 2011;110:634-41.

8. Baliatsas C, Van Kamp I, et al. Idiopathic environmental intolerance attributed to electromagnetic fields (IEI-EMF): a systematic review of identifying criteria. BMC Public Health 2012;12:643.

9. Rubin GJ, Nieto-Hernandez R, Wessely S. Idiopathic environmental intolerance attributed to electromagnetic fields (formerly 'electromagnetic hypersensitivity'): an updated systematic review of provocation studies. Bioelectromagnetics 2010;31:1-11.

10. Hausteiner C, Bornschein S, Zilker T, et al. Dysfunctional cognitions in idiopathic environmental intolerances (IEI) -an integrative psychiatric perspective. Toxicol Lett 2007;171:1-9.

11. Röösli M. Radiofrequency electromagnetic field exposure and non-specific symptoms of ill health: a systematic review. Environ Res 2008;107:277-87.

12. Johansson A, Nordin S, Heiden M, et al. Symptoms, personality traits, and stress in people with mobile phone-related symptoms and electromagnetic hypersensitivity. J Psychosom Res 2010;68:37-45.

13. Osterberg K, Persson R, Karlson B, et al. Personality, mental distress, and subjective health complaints among persons with environmental annoyance. Hum Exp Toxicol 2007;26:231-41.

14. Rubin GJ, Cleare AJ, Wessely S. Psychological factors associated with self-reported sensitivity to mobile phones. J Psychosom Res 2008;64:1-9. discussion 11-12.

15. Watson D, Pennebaker JW. Health complaints, stress, and distress: exploring the central role of negative affectivity. Psychol Rev 1989;96:234-54.

16. Roosli M, Hug K. Wireless communication fields and non-specific symptoms of ill health: a literature review. Wien Med Wochenschr 2011;161:240-50.

17. Bolger N, Davis A, Rafaeli E. Diary methods: capturing life as it is lived. Annu Rev Psychol 2003;54:579-616.

18. Shiffman S, Stone AA, Hufford MR. Ecological momentary assessment. Annu Rev Clin Psychol 2008;4:1-32.

19. Bolte JF, van der Zande G, Kamer J. Calibration and uncertainties in personal exposure measurements of radiofrequency electromagnetic fields. Bioelectromagnetics 2011;32:652-63.

20. Frei $P$, Mohler E, Burgi A, et al. Classification of personal exposure to radio frequency electromagnetic fields (RF-EMF) for epidemiological research: evaluation of different exposure assessment methods. Environ Int 2010;36:714-20.

21. Stone AA, Schwartz JE, Broderick JE, et al. Variability of momentary pain predicts recall of weekly pain: a consequence of the peak (or salience) memory heuristic. Pers Soc Psychol Bull 2005;31:1340-6.
22. Baker D, Nieuwenhuijsen MJ. Environmental epidemiology. Study methods and application. New York: Oxford University Press, 2008.

23. Van Kamp I. Project: Non-specific physical symptoms in relation to the actual and perceived exposure to EMF and the underlying mechanisms; a multidisciplinary approach. http://www.zonmw.nl/nl/ projecten/project-detail/non-specific-physical-symptoms-in-relationto-the-actual-and-perceived-exposure-to-emf-and-the-under/ samenvatting/ (accessed Mar 2013).

24. Baliatsas C, Van Kamp I, Yzermans J, et al. Non-specific physical symptoms (NSPS) and electromagnetic field (EMF) exposure: the role of perceived environmental sensitivity. Psychol Health 2011;26 (Suppl 2):6-72.

25. Stone AA, Shiffman S, Schwartz JE, et al. Patient non-compliance with paper diaries. BMJ 2002;324:1193-4.

26. Houtveen JH, Hamaker EL, Van Doornen LJ. Using multilevel path analysis in analyzing 24-h ambulatory physiological recordings applied to medically unexplained symptoms. Psychophysiology 2010;47:570-8.

27. Van der Zee KI, Sanderman R. Het meten van de algemene gezondheidstoestand met de RAND-36: een handleiding. Tweede herziene druk. Groningen: UMCG / Rijksuniversiteit Groningen, Research Institute SHARE 2012

28. Schooneveld H, Kuiper J. Electrohypersensitivity (EHS) in the Netherlands. A Questionnaire survey. 2nd graphical edition. 2008. http://www.stichtingehs.nl/images/stories/EHS/ehs_in_the_ netherlands_2008.pdf

29. Wald FD, Mellenbergh GJ. De verkorte versie van de Nederlandse vertaling van de Profile of Mood States (POMS)/the shortened version of the Dutch translation of the Profile of Mood States (POMS). Ned Tijdschr Psycholog Grensgebieden 1990;45:86-90.

30. Houtveen JH, van Doornen LJ. Medically unexplained symptoms and between-group differences in 24-h ambulatory recording of stress physiology. Biol Psychol 2007;76:239-49.

31. Roosli M, Frei P, Bolte $\mathrm{J}$, et al. Conduct of a personal radiofrequency electromagnetic field measurement study: proposed study protocol. Environ Health 2010:9:23.

32. Bolte JF, Eikelboom T. Personal radiofrequency electromagnetic field measurements in the Netherlands: exposure level and variability for everyday activities, times of day and types of area. Environ Int 2012;48:133-42.

33. Gelman A, Hill J. Multilevel power calculation using fake-data simulation. Data analysis using regression and multilevel/hierarchical models. New York: Cambridge University Press, 2007.

34. Houtveen JH, Oei NY. Recall bias in reporting medically unexplained symptoms comes from semantic memory. J Psychosom Res 2007;62:277-82.

35. Eltiti S, Wallace D, Zougkou K, et al. Development and evaluation of the electromagnetic hypersensitivity questionnaire. Bioelectromagnetics 2007;28:137-51.

36. Rosmalen JG, Wenting AM, Roest AM, et al. Revealing causal heterogeneity using time series analysis of ambulatory assessments: application to the association between depression and physical activity after myocardial infarction. Psychosom Med 2012;74:377-86.

37. Benjamini $\mathrm{Y}$, Hochberg $\mathrm{Y}$. Controlling the false discovery rate: a practical and powerful approach to multiple testing. $J R$ Statist Soc $B$ 1995;57:289-300. 